Chirurg 2016 · 87:177-178

DOI 10.1007/s00104-016-0160-y

Online publiziert: 10. Februar 2016

(c) Springer-Verlag Berlin Heidelberg 2016

\title{
CrossMark
}

Technische Innovationen bestimmen unseren Alltag. Sie verschieben Grenzen, vereinfachen Arbeitsschritte und erleichtern die Kommunikation. So haben sich auch die Grenzen in der Chirurgie verschoben. Moderne Operationstechniken haben Einzug in unsere Operationssäle gehalten. Zugänge wurden minimiert. Das Operationstrauma hat sich verringert. Damit haben sich die operativen Möglichkeiten deutlich erweitert und bei unseren Patienten neue Hoffnungen auf Heilung geweckt.

Die frühzeitige Diagnose von Krankheitsprozessen mittels hochauflösender bildgebender Verfahren, die Vertiefungen des Krankheitsverständnisses durch die molekulare Grundlagenforschung und die daraus resultierenden neuen Therapieansätze verbessern die Prognose unserer Patienten. Technische Innovationen in der minimalinvasiven Chirurgie, vielversprechende Erkenntnisse und Erfahrungen in der Roboterchirurgie eröffnen neue operative Therapiemöglichkeiten. Neue Implantatetechnologien erweitern das Spektrum des Gewebeersatzes. Der Forderung nach Gewebeschonung und -erhalt stehen komplexe radikale Therapiestrategien gegenüber, die das Überleben der Patienten verlängern sollen. Die Erwartungen der Patienten wachsen. Aber auch die Ängste nehmen zu. Patientenverfügungen sind an der Tagesordnung. Die oberste Prämisse ist, die Lebensqualität zu gewährleisten. Unsere Patienten wollen einen Lebenserhalt - aber nicht um jeden Preis.

Der diesjährige Kongress der Deutschen Gesellschaft für Chirurgie (DGCH) steht unter dem Motto: Chirurgie im Spannungsfeld von Technik, Ethik und Ökonomie. In der zum Kongress erscheinenden Sonderausgabe von Der

\section{G. Schackert}

Klinik und Poliklinik für Neurochirurgie, Universitätsklinikum Carl Gustav Carus

an der Technischen Universität Dresden, Dresden, Deutschland

\section{Chirurgie im Spannungsfeld von Technik, Ethik und Ökonomie}

Chirurg haben wir dies als Thema aufgenommen. Es soll ein repräsentativer Einblick in die „Brennpunkte“ verschiedener chirurgischer Disziplinen gegeben werden. In einer interdisziplinären Darstellung wird auf unterschiedliche operative Schwerpunkte eingegangen. Die technischen Möglichkeiten und Limitierungen werden aufgezeigt, die ethischen Grenzen werden diskutiert.

\section{) Intraoperative optische Systeme haben die Operationstechnik revolutioniert}

Es ist unzweifelhaft, dass die intraoperativen optischen Systeme, wie die Endoskopie, die Laparoskopie, der Ultraschall, fluoreszierende Farbstoffe, die Navigation, der HybridOperationssaal oder die intraoperative Magnetresonanztomographie unsere Operationstechnik revolutioniert haben. Die Zugangswege konnten schonender gestaltet und die Eingriffe verkleinert werden. Tumorgrenzen können farbgebend dargestellt werden und machen so die Exstirpation sicherer.

Neueste Entwicklungen in der Roboterchirurgie lassen uns davon träumen, dass operative Prozeduren durch Roboter mit wesentlich höherer Präzision, als dies einem Menschen möglich ist, vorgenommen werden könnten. Bisher sind die Einsätze limitiert. Neue Robotic-Systeme werden in den verschiedenen chirurgischen Disziplinen eingesetzt und überprüft.

In der Gefäßchirurgie haben interventionelle Verfahren offene chirurgische Techniken vielfach abgelöst. Allerdings gibt es heute bereits eine gute Studienlage, die differenziert die Vor- und Nachteile der unterschiedlichen Methoden analysiert hat und hilft, die Indikation zum jeweiligen Verfahren $\mathrm{zu}$ stellen.

Die Wirbelsäulenchirurgie hat durch neueste Implantatetechnologien einen erheblichen Zuwachs erfahren. Aktuelle Studien belegen nun erstmals mit Level-I-Evidenz die Indikation zur Instrumentierung bei definierten Krankheitsbildern. Die Wertigkeit der unterschiedlichen spinalen Implantate ist jedoch weiterhin in der Diskussion. Es wird ein Überblick gegeben, wann die einzelnen Implantate sinnvoll angewendet werden können. Zu den Komplikationen spinaler Implantate, den Risikofaktoren und dem Management revisionswürdiger Folgen wird Stellung genommen.

\section{》) Der besondere Fokus liegt auf der Oligometastasierung}

Die Onkologie wird von aufwendigsten Operationen bestimmt. Sie müssen im Kontext der systemischen Tumormanifestation kritisch diskutiert werden. Unser Artikel widmet sich der Metastasenchirurgie. Die unterschiedlichen Organmanifestationen mit ihren Therapiemöglichkeiten werden im interdisziplinären Kontext beleuchtet. Der besondere Fokus liegt auf der Oligometastasierung. Auch bei Hirnmetastasen ist eine chirurgische Therapie sinnvoll, die gefolgt von adjuvanten Therapiemaßnahmen zu Langzeitüberleben führen kann. Die zentrale Frage ist: Was ist die beste Therapie für Patienten mit Oligometastasierung bez. Lebensverlängerung und Lebensqualität?

Von besonderer Bedeutung ist der Stellenwert der Chirurgie in der Palliativ- 
situation. Was ist möglich, was ist sinnvoll? Das Ziel der Chirurgie in der palliativen Situation besteht vor allem in der Linderung tumorassoziierter Symptome und Komplikationen. Damit soll eine Verbesserung der Lebensqualität verbunden mit einer Lebenszeitverlängerung herbeigeführt werden. Die Evidenzlage ist allerdings sehr schwach, da randomisierte Studien in dieser Situation ethisch angreifbar sind.

Der Anspruch auf technische Perfektion und ethische Verantwortung dem Patienten gegenüber beinhaltet die Grundsäulen des ärztlichen Handelns. Eine interdisziplinäre, auf das Wohl des Patienten ausgerichtete Therapie unter Berücksichtigung der Grenzen des Machbaren wird diesem Anspruch gerecht. Ökonomische Vorgaben werden zwangsläufig dem Gleichklang von technischem und ethischem Anspruch zuwider laufen.

Die vorliegende Kongressausgabe von Der Chirurg soll einen Einstieg in unsere Kongressthemen bieten. Wir erwarten in Berlin einen offenen und konstruktiven Dialog.

Wir freuen uns auf Ihr Kommen.<smiles>CCCC</smiles><smiles>CCCCCCCC(C)(C)C</smiles>

Prof. Dr. Gabriele Schackert Präsidentin der Deutschen Gesellschaft für Chirurgie

\section{Korrespondenzadresse}

\section{Prof. Dr. G. Schackert}

Klinik und Poliklinik für Neurochirurgie, Universitätsklinikum Carl Gustav Carus an der Technischen Universität Dresden Fetscherstraße 74, 01307 Dresden Gabriele.Schackert@uniklinikum-dresden.de

Interessenkonflikt. G. Schackert gibt an, dass kein Interessenkonflikt besteht.

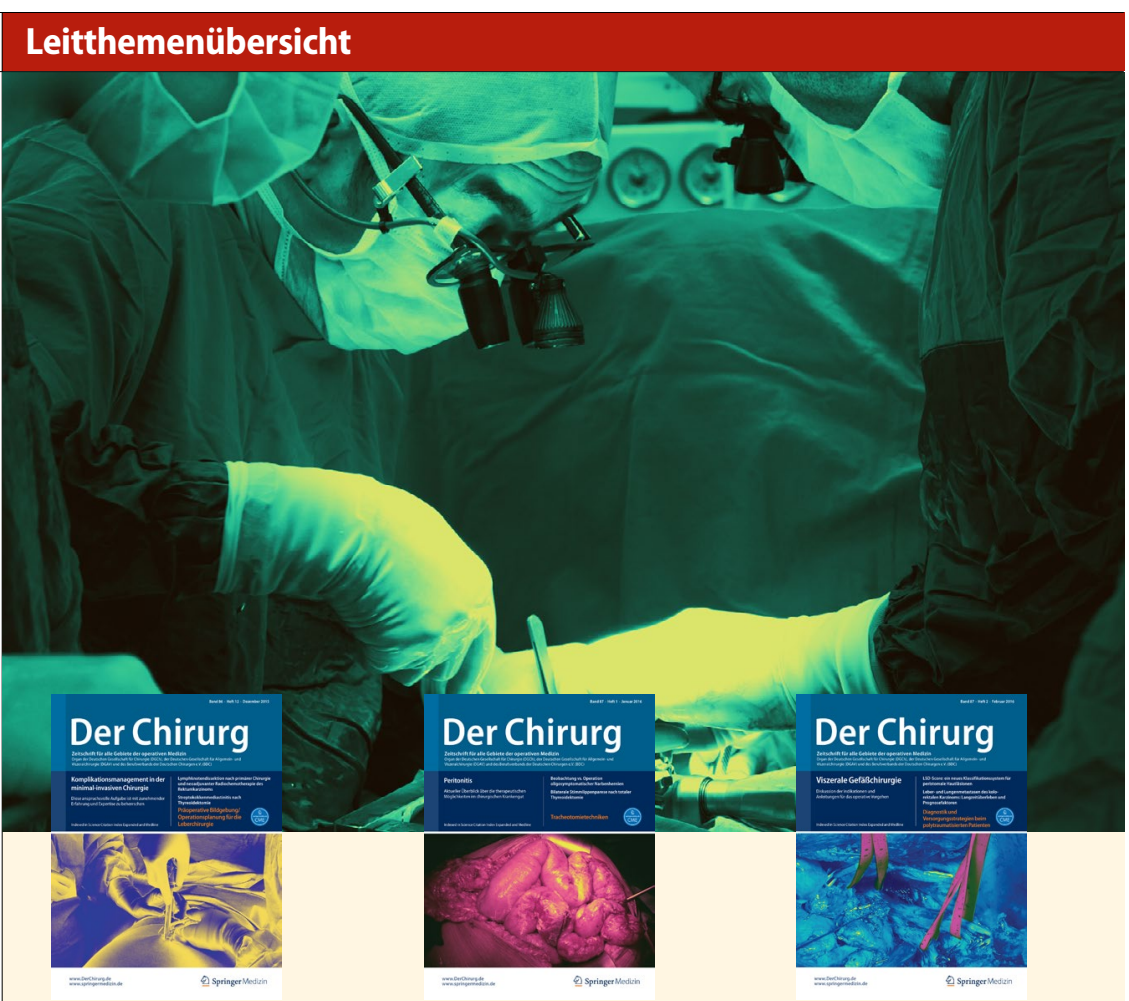

Die Zeitschrift Der Chirurg bietet Ihnen jeden Monat umfassende und aktuelle Beiträge zu interessanten Themenschwerpunkten aus allen Bereichen der Chirurgie.

\section{Überblick 2015}

01/15 Komplikationen in der endokrinen Chirurgie

02/15 Komplikationen in der Leberchirurgie

03/15 Plastisch-rekonstruktive Chirurgie und Präfabrikation von Geweben im interdisziplinären Komplikationsmanagement

04/15 Komplikationsmanagement des unteren Gastrointestinaltraktes

05/15 Komplikationen in der Thoraxchirurgie

06/15 Komplikationen in der hepatopankreato-biliären Chirurgie

07/15 Komplikationen nach gefäßchirurgischen Interventionen

08/15 Komplikationsmanagement in der proktologischen Chirurgie

09/15 Komplikationsmanagement Adipositaschirurgie

10/15 Komplikationen in der Unfallchirurgie nach Operationen am oberen Gastrointestinaltrakt

12/15 Komplikationsmanagement in der minimal-invasiven Chirurgie
11/15 Management von Komplikationen

\section{Vorschau 2016}

01/16 Peritonitis

02/16 Viszerale Gefäßchirurgie

03/16 Chirurgie im Spannungsfeld von Technik, Ethik und Ökonomie

04/16 Gastroduodenale und rektale neuroendokrine Neoplasien

05/16 Erweiterung des chirurgischen Spektrums durch neue onkologische Therapie

06/16 Mediastinitis

07/16 Rektumkarzinom, offen oder laparoskopisch?

08/16 Roboterchirurgie in der Viszeralchirurgie

09/16 Zugangswege und Bauchdeckenverschluss

10/16 Implantat-assoziierte Infektionen in Orthopädie und Unfallchirurgie

11/16 Klammernahtoperationen am Anorektum - Indikationen, Techniken, Ergebnisse

12/16 OP-Saal der Zukunft
Alle Inhalte der Zeitschrift Der Chirurg finden Sie unter www.springermedizin.de/der-chirurg SpringerMedizin.de bietet Ihnen Zugang zu allen elektronisch verfügbaren Ausgaben Ihrer Zeitschrift - unabhängig davon, seit wann Sie die Zeitschrift abonniert haben.

Unser Tipp: Mit dem e.Med Kombi-Abo können Sie jederzeit auf alle Inhalte von SpringerMedizin.de zugreifen. Auf Wunsch erhalten Sie darüber hinaus eine gedruckte Fachzeitschrift Ihrer Wahl.

Testen Sie e.Med 30 Tage lang kostenlos und unverbindlich!

Jetzt informieren unter www.springermedizin.de/eMed oder telefonisch unter 0800-77 80777 (Montag bis Freitag, 10 bis 17 Uhr) 